\title{
Equipes de psychiatrie mobiles
}

\section{Stéphane Morandi}

MD et maître d'enseignement et de recherche; médecin associé, Service de psychiatrie communautaire, Département de psychiatrie du CHUV; médecin cantonal adjoint, Service de la santé publique, Département de la santé et de l'action sociale du canton de Vaud

\section{Introduction}

En Suisse, comme dans de nombreux pays occidentaux, le mouvement de désinstitutionalisation qui a frappé la psychiatrie durant la seconde moitié du $\mathrm{XX}^{\mathrm{e}}$ siècle a conduit à la fermeture de nombreux lits psychiatriques ( $-44 \%$ entre 1992 et 2011) [1]. Contrairement à d'autres pays et à l'exception de quelques initiatives locales, cette tendance n'a pas été accompagnée par le développement de la psychiatrie communautaire. Celle-ci vise à proposer, dans un périmètre géographique défini, des soins qui répondent aux besoins de la population locale, à mettre en lien les services offrant des prestations sanitaires et sociales et à les rendre accessibles aux personnes qui en ont besoin [2]. Une attention particulière est portée aux facteurs relationnels, sociaux et environnementaux qui déterminent la santé mentale. Cet article présente les équipes de psychiatrie mobiles, pilier de la psychiatrie communautaire, avance certaines hypothèses pouvant expliquer leur développement marginal en Suisse et propose des pistes qui permettraient d'encourager ce type d'approche.

\section{Zusammenfassung}

Wie in zahlreichen anderen Ländern der westlichen Welt führte die in der zweiten Hälfte des 20. Jahrhunderts in der Psychiatrie aufgekommene Desinstitutionalisierungsbewegung auch in der Schweiz zu einem Wegfall zahlreicher Betten im psychiatrischen Bereich (- 44 \% zwischen 1992 und 2011) [1]. Anders als in anderen Ländern und mit Ausnahme kleinerer lokaler Initiativen wurde dieser Trend jedoch nicht durch die Ausweitung einer gemeindenahen psychosozialen Versorgung begleitet. Letztere bietet eine bedarfsgerechte, genau auf die Menschen einer bestimmten Region zugeschnittene Pflege, verknüpft sanitäre und soziale Leistungsangebote und macht sie jenen Menschen zugänglich, die sie benötigen [2]. Dabei liegt ein besonderes Augenmerk auf den relationalen, sozialen und umweltspezifischen Faktoren, die mentale Gesundheit ausmachen. Dieser Artikel stellt die mobilen psychiatrischen Einsatzteams als Säulen einer solchen gemeindenahen Versorgung vor, bietet mögliche Ursachen für ihre marginale Entwicklung in der Schweiz und schlägt Mittel und Wege zur Förderung dieses Therapieansatzes vor.

\section{Les équipes de psychiatrie mobiles}

On estime généralement que la transformation de l'asile psychiatrique en hôpital de soins aigus a été profitable à la majorité des patients. Cependant, certains groupes d'individus souffrant de troubles sévères ou accumulant les difficultés sur le plan psychique, physique et social n'ont pas bénéficié de ces changements de paradigme. Tout d'abord, dans un système de santé qui ne cesse de se spécialiser, les chances de recevoir des soins diminuent à mesure que la complexité de la situation d'une personne augmente, car chaque nouveau besoin constitue un critère d'exclusion potentiel [3]. Ainsi, une partie des patients qui en ont le plus besoin échappent aux soins et se retrouvent en situation de grande précarité. Une étude menée à Zurich en 2012 a démontré qu'une majorité des personnes vivant dans les lieux d'hébergement d'urgence de la ville souffraient de psychopathologies et la sévérité de leurs difficultés dépassait souvent celle des patients hospitalisés en psychiatrie [4]. Ensuite, plutôt que de pouvoir vivre dans la communauté, certaines personnes ont simplement été transférées de l'hôpital vers d'autres structures, telles qu'établissements médico-sociaux, foyers ou prisons [5]. Enfin, un petit nombre de patients restent hospitalisés en psychiatrie durant de longues périodes, sans alternative envisageable, ou sont contraints d'être réadmis très régulièrement et consomment une part importante de l'offre de soins [6]. Le retour dans la communauté de certains patients a parfois fait peser une charge importante sur les proches et les prestataires de soins de premier recours, qui ne disposent pas toujours des ressources nécessaires pour faire face aux situations les plus complexes [7].

Pour répondre à ces difficultés, des équipes de psychiatrie mobiles ont été développées depuis les années soixante, d'abord dans les pays anglo-saxons, puis dans la majorité des pays occidentaux. Leur but est de permettre la vie dans la communauté de personnes souffrant de troubles psychiques sévères et de favoriser leur rétablissement à travers une approche collaborative avec le patient et les membres de son réseau, qu'ils soient proches ou professionnels, tout en limitant les prestations plus coûteuses, telles que séjours 
en hôpital psychiatrique ou visites dans les services d'urgence. A ce jour, ces équipes restent marginales en Suisse. A titre d'exemple, plusieurs équipes de psychiatrie mobiles ont été créées au sein du Département de psychiatrie du CHUV à Lausanne depuis 2001. Tout d'abord, le suivi intensif dans le milieu a été développé pour favoriser l'engagement dans les soins des personnes souffrant de troubles psychiques sévères les plus réfractaires ou les plus marginalisées, ainsi que pour optimiser le suivi des patients dit «hauts consommateurs", c'est-à-dire recourant fréquemment aux services d'urgence ou étant très régulièrement hospitalisés [8]. En 2014, l'approche a été adaptée avec succès pour les problèmes d'addiction [9]. En 2007, le case management de transition a été développé afin de faciliter le retour à domicile, après une hospitalisation en psychiatrie, pour les patients suivis par un médecin généraliste ou un psychiatre travaillant en cabinet privé [10]. Le projet pilote "housing first», en collaboration avec la Ville de Lausanne, et un foyer socio-éducatif, a permis de proposer un logement indépendant encadré par une équipe mobile à des patients qui ne parvenaient pas à accéder à des structures d'hébergement de type foyer ou EMS, par exemple en raison de consommations actives de substances, ou présentant des difficultés à vivre dans un milieu communautaire [11]. Ces différentes interventions sont toutes basées sur deux modèles théoriques. Le premier, l'assertive community treatment (ACT), propose que des équipes multidisciplinaires à même de répondre aux différents besoins du patient aillent à la rencontre de ce dernier là où il se trouve, généralement à domicile, en structure d'hébergement ou même dans la rue [12]. Chacun des intervenants suit un petit nombre de situations, afin de pouvoir être disponible, flexible et intervenir de manière intensive lorsque cela est nécessaire. L'approche collaborative, non médico-centrée, dans laquelle les objectifs du suivi sont fixés avec le patient, est grandement appréciée par ce dernier [13]. A Lausanne, le modèle a été adapté, afin de mettre un accent particulier sur les collaborations avec le réseau, particulièrement les soins de premier recours. Dans ce sens, les interventions s'inspirent d'un second modèle théorique, la critical time intervention (CTI) qui recommande un soutien aussi bien pratique que psychologique, une évaluation des compétences et des limitations de la personne en situation réelle et une intervention limitée aux moments de crise [14].

De par leur modèle d'intervention, les équipes de psychiatrie mobiles sont relativement coûteuses, notamment en termes de ressources humaines. Elles doivent donc cibler les patients qui en ont le plus besoin et ne remplacent pas les autres services psychiatriques, mais complètent l'offre de soins et optimisent le recours aux autres prestataires, en s'inscrivant dans un modèle de soins équilibré (balanced care model) [15]. Malgré un impact très favorable sur les personnes suivies et la grande satisfaction de ces dernières et de nombreux acteurs du réseau, les équipes de psychiatrie mobiles peinent à s'implanter en Suisse. Plusieurs hypothèses peuvent être avancées pour expliquer cet état de fait.

\section{Raisons pouvant expliquer le manque de développement des équipes de psychiatrie mobiles et, plus généralement, de la psy- chiatrie communautaire en Suisse}

Premièrement, la psychiatrie communautaire reste une branche méconnue de la médecine en Suisse. A titre d'exemple, à la Faculté de Biologie et de Médecine de Lausanne, les médecins en formation n'ont que deux heures sur leurs six années d'étude consacrées au sujet. Au niveau postgradué, seuls trois des 75 modules dispensés par le centre d'enseignement postuniversitaire pour la spécialisation en psychiatrie et psychothérapie (CEPUSPP) concernent la psychiatrie communautaire. Par conséquent, la grande majorité des médecins, même psychiatres, n'ont pas de connais sances ni d'expérience dans le domaine. Or, les populations concernées cumulent généralement les difficultés sur le plan psychique, physique et social et présentent des besoins complexes. S'en occuper requiert donc des compétences spécifiques.

Deuxièmement, comme l'a démontré un rapport national, la psychiatrie communautaire n'est pas suffisamment valorisée en termes économiques [16]. Une part du financement de tels programmes dépend de subsides cantonaux dont la pérennité n'est pas assurée sur le long terme. En effet, le temps consacré à engager la personne dans un suivi avant même de pouvoir ouvrir un dossier médical, les nombreux déplacements, le travail de réseau et les rendez-vous manqués souvent inéluctables lorsque l'on est confronté à ce type de population sont des actes peu valorisés ou non couverts par l'assurance-maladie du patient et requiert d'autres formes de financement. Dans ces conditions, les responsables d'institutions psychiatriques ont peu d'intérêt à développer ce type d'approches, particulièrement s'il s'agit de le faire aux dépens de lits psychiatriques plus rentables, et ce, même si, au final, elles s'avèrent être bénéfiques pour les patients et leurs proches et peuvent soulager un système de soins souvent engorgé.

Finalement, la Suisse fait face à un haut taux de recours à la contrainte dans les soins psychiatriques [17]. Pour 
certains, ces chiffres démontrent la qualité d'un système qui ne laisse personne sur le carreau. Cependant, ils peuvent aussi refléter le fait que, confrontés à des patients se trouvant en situation de crise, les médecins ont tendance à décider d'une hospitalisation, parfois faute de solution pour une prise en charge à domicile, mais aussi pour éviter de devoir répondre légalement d'un accident ou d'un suicide, plutôt que de laisser une personne souffrant de trouble psychique à domicile, et ce, même avec le soutien d'une équipe de psychiatrie mobile.

\section{Pistes pouvant favoriser le renforce- ment et le développement des équipes de psychiatrie mobiles}

Contrairement à d'autres pays, tels que le RoyaumeUni, la Hollande, l'Allemagne ou la Norvège, jusqu'ici, la Suisse n'a pas mis en place de programme national visant à développer la psychiatrie communautaire. En l'absence de politique nationale sur le sujet, le développement de réseaux cantonaux et transcantonaux réunissant les professionnels travaillant dans le champ de la psychiatrie communautaire pourrait permettre le partage d'expériences, l'échange de connaissances et une meilleure représentation auprès des autorités politiques. Le mode de financement des équipes de psychiatrie mobiles devrait également être reconsidéré avec, par exemple, la mise en place d'un forfait journalier ou l'introduction d'un financement par cas plutôt que par service pour les situations complexes pouvant bénéficier de ce type d'approche. Le développement d'alternatives à l'hospitalisation pourrait être soutenu sur le plan politique. Enfin, la formation des professionnels concernant la psychiatrie communautaire devrait être développée aux niveaux pré- et postgrades.

\section{Conclusion}

Les équipes de psychiatrie mobiles représentent une piste intéressante pour le maintien à domicile et le rétablissement des personnes souffrant de troubles psychiques sévères. Elles permettent également d'envisager une réduction des coûts de la santé liés à ces pathologies. D'autres formes d'interventions méritent encore d'être testées dans le contexte suisse. Les équipes de psychiatrie mobiles d'intervention de crise qui interviennent dans l'urgence permettraient notamment de limiter le recours aux hospitalisations et à la contrainte [18].
Références

$1 \mathrm{H}+$. Les Grandes tendances de la psychiatrie 2017. Available from http://www.hplus.ch/fr/chiffres_faits/moniteur_des_hopitaux_ et_cliniques_de_h/psychiatrie/

2 Thornicroft G, Szmukler G. Textbook of Community Psychiatry. Oxford: Oxford University Press; 2001.

3 Hwang SW, Burns T. Health interventions for people who are homeless. Lancet. 2014;384.

4 Jaeger M, Briner D, Kawohl W, Seifritz E, Baumgartner-Nietlisbach G. Psychosocial functioning of individuals with schizophrenia in community housing facilities and the psychiatric hospital in Zurich. Psychiatry Research. 2015;230(2):413-8.

5 Priebe S, Badesconyi A, Fioritti A, Hansson L, Kilian R, TorresGonzales F, et al. Reinstitutionalisation in mental health care: comparison of data on service provision from six European countries. BMJ. 2005;330(7483):123.

6 Golay P, Morandi S, Conus P, Bonsack C. Identifying patterns in psychiatric stays: towards a typology of post-desinstitutionalisation hospitalisation trajectories. Manuscript in preparation. 2018.

7 Saxena S, Thornicroft G, Knapp M, Whiteford H. Global Mental Health 2 - Resources for mental health: scarcity, inequity, and inefficiency. Lancet. 2007;370(9590):878-89.

8 Bonsack C, Adam L, Haefliger T, Besson J, Conus P. Difficult-to-engage patients: A specific target for time-limited assertive outreach in a Swiss setting. Can J Psychiatry - Revue Canadienne De Psychiatrie. 2005;50.

9 Morandi S, Silva B, Golay P, Bonsack C. Intensive Case Management for Addiction to promote engagement with care of people with severe mental and substance use disorders: an observational study. Substance Abuse Treatment, Prevention, and Policy. 2017;12(1):26.

10 Bonsack C, Golay P, Manetti SG, Gebel S, Ferrari P, Besse C, et al. Linking Primary and Secondary Care after Psychiatric Hospitalization: Comparison between Transitional Case Management Setting and Routine Care for Common Mental Disorders. Frontiers in Psychiatry. 2016 Jun;7.

11 Garcia Gonzalez De Ara C, Morandi S, Lippuner M, Delmatti J, Golay P, Bonsack C. «Chez soi d'abord»: se rétablir chez soi d'un trouble psychique sévère. Revue Médiale Suisse. 2017;13:1605-9.

12 Burns T, Firn M. Oxford: Oxford University Press. 2002.

13 Priebe S, Watts J, Chase M, Matanov A. Processes of disengagement and engagement in assertive outreach patients: qualitative study. British Journal of Psychiatry. 2005;187:438-43.

14 Herman DB. Transitional Support for Adults With Severe Mental Illness: Critical Time Intervention and Its Roots in Assertive Community Treatment. Research on Social Work Practice. 2014;24(5):556-63.

15 Thornicroft G, Tansella M. The balanced care model: the case for both hospital- and community-based mental healthcare. British Journal of Psychiatry. 2013;202(4):246-8.

16 Stocker D, Jäggi J, Legler V, Künzi K. Critère de réussite des services de psychiatriques mobiles. Berne: Office fédéral de la santé publique, 2018.

17 Schuler D, Tuch A, Peter C. Placement en établissement psychiatrique à des fins d'assistance. Neuchâtel: Observatoire suisse de la santé, 2018.

18 Wheeler C, Lloyd-Evans B, Churchard A, Fitzgerald C, Fullarton K, Mosse L, et al. Implementation of the Crisis Resolution Team model in adult mental health settings: a systematic review. BMC Psychiatry. 2015/04/08;15(1):74.
Correspondance:

Dr Stéphane Morandi Consultations de Chauderon Place Chauderon 18 CH-1003 Lausanne Tél. 0213140050 stephane.morandi[at] chuv.ch 